\section{The sensory immune system: a neural twist to the antigenic discontinuity theory}

\author{
Serge Nataf
}

In their recent Essay (The speed of change: towards a discontinuity theory of immunity? Nature Rev. Immunol. 13, 764-769 (2013)) ${ }^{1}$, Pradeu et al. proposed that rather than antigens per se, antigenic discontinuity is a major determinant of immune responses. However, the immune system is currently viewed as a complex network of cells and molecules that are endowed with the unique ability to discriminate self from non-self and to recognize specific molecular patterns associated with pathogens or damaged tissues $^{2-5}$. To reconcile those two partially divergent theories, I argue that our current theoretical conception of immunity is possibly biased by an incomplete picture of the so-called 'immune recognition' process. Indeed, although Jerne ${ }^{6}$ was the first to enrich the immunological lexicon with neuroscience-derived terms, such as 'recognition', 'learning' and 'memory' (REFS 4,6), this analogy-based reasoning may have missed some important aspects of neurophysiology and neurocognitive science.

This observation may prove to be of particular interest when considering sensory perception as the neural counterpart of immune recognition. In the nervous system, sensory signals captured by specific peripheral receptors are transmitted to the central nervous system and are eventually integrated in specific cortical areas of the brain before an adapted reaction and/or a memory trace is or is not generated. Sensory perception is thus supported by two distinct processes: one, signal reception; and two,

\section{a The sensory nervous system}

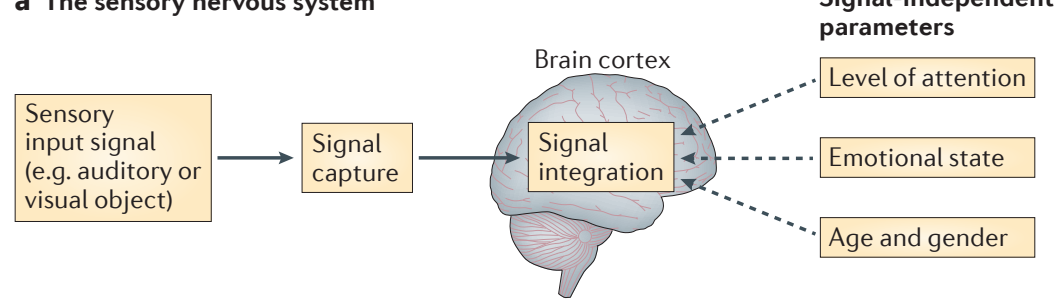

b The sensory immune system

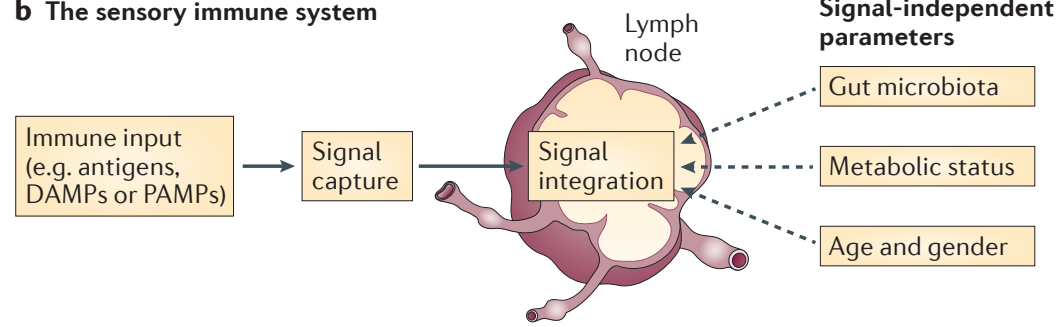

Figure 1 Comparison of the sensory nervous and immune systems. a|A schematic representation of the sensory nervous system. In the sensory nervous system, a sensory input, such as an auditory or visual object, is first captured by specialized neurons. The captured signal is then transmitted towards and integrated in specific brain cortical areas in which specialized neuronal cell populations reside and interact. Such an integration step is shaped by signal-independent parameters, including the age, gender, level of attention and the emotional state of the host. $\mathbf{b} \mid$ A schematic representation of the sensory immune system. In the proposed view of a sensory immune system, any immune input (for example, antigens, danger-associated molecular patterns (DAMPs) or pathogen-associated molecular patterns (PAMPs)) is first captured by specialized immune cells, in particular, but not exclusively, by antigen-presenting cells. The captured signal is then transmitted towards and integrated in the lymph nodes or other secondary lymphoid organs. There, the integration step relies on complex cell cooperation that is shaped by signal-independent parameters. These parameters include the age, gender, metabolic status and the gut microbiota composition of the host. signal integration. Interestingly, brain cortical integration of sensory signals is dictated by a number of parameters that are signal-independent and may fluctuate over time. These include, in particular, our level of consciousness and attention, our emotional state, and the superimposition of distinct input signals, not to mention our age and gender (FIG. 1a).

As an example, depending on our auditory attention and the level of background noise accompanying a given auditory input, the auditory processing (integration) step may or may not permit the discrimination of meaningful auditory objects from meaningless ones $^{7,8}$. Also, although the rhythmicity of an auditory input is one of a number of signaldependent features that drives our perception of this particular signal, this perception may be in fine shaped by signal-independent factors that comprise our auditory attention and by the presence of superimposed auditory inputs ${ }^{9-11}$. On this basis, a parallel could be established between the sensory nervous system and the afferent arm of immunity, and we propose the term 'immune perception' to describe the function of 'immune recognition'. In this conception of a sensory immune system, perception of immune signals would not only be determined by their intrinsic nature (for example, their antigenic structure, and the presence of danger-associated molecular patterns (DAMPs) or pathogenassociated molecular patterns (PAMPs)) and the rhythmicity of their occurrence (antigenic discontinuity) but also by signal-independent parameters that would condition the integration step of immune perception. These would notably include age ${ }^{12}$, gender ${ }^{13}$, metabolic status $^{14}$ and gut microbiota composition ${ }^{15}$. In all likelihood, such an integration step would essentially take place in secondary lymphoid organs and would be shaped by systemic blood-circulating factors (FIG. 1 b).

At a time when the large and fascinating field of theoretical immunology is (re)opened to non-immunologists ${ }^{1}$, I suggest that neuroscientists might be helpful for rediscovering and refining the parallel between the nervous and the immune systems that was initially highlighted by Jerne ${ }^{6}$.

Serge Nataf is at the Lyon Neuroscience Research Center, INSERM 1028CNRS UMR5292, University

Lyon-1, Banque de tissus et de cellules, Hôpital Edouard Herriot, Lyon University Hospital (Hospices Civils de Lyon), Lyon F-69000, France.

e-mail: serge.nataf@inserm.fr http://dx.doi.org/10.1038/nri3521-c1

1. Pradeu, T., Jaeger, S. \& Vivier, E. The speed of change: towards a discontinuity theory of immunity? Nature Rev. Immunol. 13, 764-769 (2013).

2. Burnet, F. M. \& Fenner, F. The Production of Antibodies 2nd edn (Macmillan and Co., 1949). 
CORRESPONDENCE

3. Tauber, A. I. The Immune Self: Theory or Metaphor? (Cambridge Univ. Press, 1994).

4. Tauber, A. in The Stanford Encyclopedia of Philosophy (ed. Zalta, E. N.) [online], http://plato. stanford.edu/archives/sum2012/entries/biology-self/ (2012).

5. Matzinger, P. The danger model: a renewed sense of self. Science 296, 301-305 (2002).

6. Jerne, N. K. Towards a network theory of the immune system. Ann. Immunol. (Paris) 125C, 373-389 (1974).

7. Rabinowitz, N. C., Willmore, B. D., King, A. J. \& Schnupp, J. W. Constructing noise-invariant representations of sound in the auditory pathway. PLoS Biol. 11, e1001710 (2013).

8. Bar-Yosef, O., Rotman, Y. \& Nelken, I. Responses of neurons in cat primary auditory cortex to bird chirps: effects of temporal and spectral context. J. Neurosci. 22, 8619-8632 (2002).

9. Lakatos, P. et al. The spectrotemporal filter mechanism of auditory selective attention. Neuron 77, 750-761 (2013).

10. Okamoto, H., Teismann, H., Keceli, S., Pantev, C. \& Kakigi, R. Differential effects of temporal regularity on auditory-evoked response amplitude: a decrease in silence and increase in noise. Behav. Brain Funct. 9, 44 (2013).

11. Seydell-Greenwald, A., Greenberg, A. S. \&

Rauschecker, J. P. Are you listening? Brain activation associated with sustained nonspatial auditory attention in the presence and absence of stimulation. Hum. Brain Mapp. http://dx.doi.org/10.1002 hbm.22323 (2013).

12. Anderson, C. C. \& Matzinger, P. Immunity or tolerance: opposite outcomes of microchimerism from skin grafts. Nature Med. 7, 80-87 (2001).

13. Zhao, G., Moore, D. J. \& Kim, J. I. Inhibition of transplantation tolerance by immune senescence is reversed by endocrine modulation. Sci. Transl Med. 3. 87ra52 (2011).

14. Procaccini, C., De Rosa, V. \& Galgani, M. An oscillatory switch in mTOR kinase activity sets regulatory $\mathrm{T}$ cell responsiveness. Immunity 33, 929-941 (2010).

15. lida, N., Dzutsev, A. \& Stewart, C. A Commensal bacteria control cancer response to therapy by modulating the tumor microenvironment. Science 342, 967-970 (2013)

Competing interests statement

The author declares no competing interests. 